\title{
Analysis on the Treatment of Trimetazidine Jointed with Metoprolol in the Coronary Heart Disease with the Heart Failure
}

\author{
Lingdong Meng ${ }^{1, a}$, Yuntao Cheng, ${ }^{2, *}$ \\ ${ }^{1}$ Ruyi Jia11. Department of Cardiology, Shandong Province Ji'nan 4th People's Hospital, Ji'nan \\ 250031, China \\ ${ }^{2}$ Department of Cardiology, Affiliated hospital of Jining Medical University , Ji'nan 272000, China \\ amengmeng@126.com
}

*Corresponding author cheng678@126.com

Keywords: Coronary heart disease (CHD); Heart failure; Trimetazidine; Metoprolol

\begin{abstract}
Objective: To observe and analyze the curative effect of the Trimetazidine joint with Metoprolol in the patients with heart failure and coronary heart disease, the treatment of patients with coronary heart disease with heart failure to provide certain reference. Methods: From May 2013 to May 2014, 76 cases of coronary heart disease with heart failure patients are divided into two groups by random method. Two groups of patients were given comprehensive treatment, the control group 38 patients on the basis of the given Trimetazidine treatment, treatment group on the basis of Trimetazidine with Metoprolol therapy. Results: After treatment, the treatment group therapy effect and improve cardiac function were significantly better than that in control group $(\mathrm{P}<$ 0.05). Conclusions: In patients with coronary heart disease with heart failure on the basis of conventional treatment with Trimetazidine joint with Metoprolol, the function and effect is very good, and is worthy of clinical popularization and application.
\end{abstract}

\section{Introduction}

Heart failure or heart failure gratified the function is not complete, always called congestive heart failure. It is caused by the changes of cardiac structure and function, finally led to a decline in heart pump function, pulmonary and systemic blood, can't satisfy the need of tissue metabolism. The signs and symptoms of heart failure are fatigue, asthma, the increased heart rate, cardiac hypertrophy, etc. In the terminal stage of various heart disease, heart failure is a common clinical syndrome, a group of also is one of the common causes of coronary heart disease, coronary heart disease (CHD) heart failure patients with serious threat to the life and health, so positive and effective treatment to reduce mortality and improve patients prognosis is of great importance. Selection in May 2013 to May 2014, 76 patients with coronary heart disease with heart failure, treatment, now report as follows.

\section{Materials and Methods}

\section{Materials}

We select 76 cases of coronary heart disease with heart failure patients in May 2013 to May 2014, and they are randomly divided into two groups, treatment group ( 38 cases ( 22 male, female 16 cases. $54 \sim 76$ years old, average 65.2 years; Duration of $2 \sim 4$ a, an average of $3.2 \mathrm{~b} 23$ cases of the control group of 38 cases of male, female 15 cases. $53 \sim 75$ years old, average 64.8 years old; Duration of $2 \sim 4 \mathrm{c}$, an average of $3.3 \mathrm{~d}$, conforms to the WHO criteria for the diagnosis of coronary heart disease (CHD) heart failure. Two groups of patients are no significant differences in age, sex and disease, comparable. 


\section{The treatment methods}

Two groups of patients were given cardiotonic agent. ACEI and ARB and other comprehensive treatment. The treatment group is with Metoprolol and Trimetazidine jointed. Metoprolol 2 times/d, starting dose of $6.25 \mathrm{mg} / \mathrm{d}$, according to the clinical manifestations of gradually increase the dose, the biggest target dose not exceed $100 \mathrm{mg} / \mathrm{d}$; Trimetazidine $2 \sim 3$ times $/ \mathrm{d}, 20 \mathrm{mg}$ a day. The control group only give Trimetazidine treatment, to observe the therapeutic effect of patients of two groups and to improve cardiac function.

\section{Statistical processing}

With mathematical statistics software SPSS19.0 for data collection and statistical analysis, and chi-square test, $\mathrm{P}<0.05$, significant difference has statistical significance.

\section{Results}

After treatment, the treatment group therapy effect and improve cardiac function were significantly better than that in control group $(\mathrm{P}<0.05)$.

Table 1: The curative effect comparison between two groups

\begin{tabular}{cccccc}
\hline Group & $\mathrm{n}$ & $\begin{array}{c}\text { Significantly } \\
\text { effective }\end{array}$ & Effective & Invalid & $\begin{array}{c}\text { Total } \\
\text { effective rate }\end{array}$ \\
\hline $\begin{array}{c}\text { The } \\
\text { treatment }\end{array}$ & 38 & $27(71.06)$ & $9(23.68)$ & $2(5.26)$ & 94.74 \\
$\begin{array}{c}\text { group } \\
\text { The control } \\
\text { group }\end{array}$ & 38 & $18(47.37)$ & $14(36.84)$ & $6(15.79)$ & 84.21 \\
$\quad \chi 2$ & & & & & 8.065 \\
P value & & & & & $<0.05$ \\
\hline
\end{tabular}

Table 2: The two groups after treatment related index statistics

\begin{tabular}{ccccccc}
\hline Group & $\mathrm{n}$ & $\begin{array}{c}\text { Heart } \\
\text { rate } \\
/ \mathrm{min}\end{array}$ & $\begin{array}{c}\text { Systolic } \\
\text { blood } \\
\text { pressure } \\
/ \mathrm{mmHg}\end{array}$ & LVEF/\% & LVESD/mm & LVEDD/mm \\
\hline $\begin{array}{c}\text { The } \\
\text { treatment } \\
\text { group }\end{array}$ & 38 & $82.7 \pm 3.3$ & $128.8 \pm 5.6$ & $52.2 \pm 2.8$ & $32.8 \pm 2.6$ & $53.7 \pm 3.6$ \\
$\begin{array}{c}\text { The } \\
\text { control } \\
\text { group }\end{array}$ & 38 & $80.3 \pm 3.1$ & $124.7 \pm 5.1$ & $45.6 \pm 3.8$ & $38.8 \pm 2.7$ & $61.2 \pm 4.6$ \\
$\chi 2$ & & 6.794 & 7.025 & 8.972 & 7.053 & 9.145 \\
P value & $<0.05$ & $<0.05$ & $<0.05$ & $<0.05$ & $<0.05$ \\
\hline
\end{tabular}

\section{Discussion}

According to the world health organization (WHO), the crowd incidence of CHF is $1.3 \% \sim 5.6 \%$. With the increase of age, the emergence of diseases, the incidence of CHF will gradually rise, and that means higher mortality and disability. At present, the treatment of patients with heart failure of coronary (CHD) is mainly to improve the blood supply of coronary artery and blood flow dynamics is given priority to, conventional drug in improving the clinical symptoms and cardiac function in patients, all good curative effect, improve the survival quality of patients, reducing the case fatality rate, and reducing the risk of cardiovascular events. Based on the pathophysiology of heart failure occurred ventricular remodeling and myocardial fibrosis. Intervening in the myocardial energy 
metabolism, mitochondrial energy metabolism process is perfect, and then achieves the goal of treatment of CHF. As a relatively new regulation of myocardial cell metabolism of drugs, Trimetazidine and Myocardial energy metabolism can be optimized to reduce myocardial ischemia, improving myocardial function, increase the purpose of glucose metabolism, and improve cardiac function and decrease the damage degree.

\section{The clinical manifestations of coronary heart disease}

The coronary heart disease is coronary atherosclerosis and vascular obstruction, which leads to myocardial ischemia, hypoxia, or coronary artery functional change. Long-term myocardial ischemia, hypoxia and myocardial tissue nutrition disorder heart failure eventually lead to the deterioration of cardiac function. Conventional treatments for homodynamic effects of drugs, the treatment principle is mainly through improving aerobic capacity, rather than through improving the myocardial cell energy metabolism and function, so the curative effect of routine comprehensive treatment is not ideal.

The coronary heart disease (CHD) is a kind of more common cardiovascular disease, the disease development to the end, may cause various complications, such as heart failure, myocardial infarction, a serious threat to life and health of patients. Including coronary heart disease ,heart failure are caused by coronary heart disease, cardiovascular happening, hardening and pathological changes, cause not oxygen in time, myocardial cells appear enough oxygen, and myocardial cell aging lesions occur, cells cannot be conducted internal energy metabolism, leading to loss of heart contractions. Compliance is abate, causing complications such as heart failure or arrhythmia. Traditional medicines to treat the disease is mainly for the homodynamic effects of drugs, can through reducing the peripheral resistance, increasing coronary blood flow and collateral circulation, reducing myocardial oxygen consumption, etc., it improves ventricular diastolic and systolic function. Traditional medicine treatment is based on the improvement of the aerobic capacity, rather than to improve the myocardial cell energy metabolism play its efficacy, for elderly patients with vascular lesions in general is relatively serious, its treatment effect is not ideal.

Heart attack lead examination showed mainly R wave, ST segment down, flat or inverted T wave attack recover gradually after several minutes. Electrocardiogram changes of patients, can consider doing electrocardiogram test. Attack is not typical, diagnosis depends on observing the curative effect of nitroglycerin and electrocardiogram changes during the attack, or the dynamic electrocardiogram for $24 \mathrm{~h}$ continuous monitoring. The diagnosis has difficulty, feasible, MDCT radioactive nuclide myocardial imaging or MRI, such as is necessary to consider lines of selective coronary angiography. With more than $\mathrm{X}$ syndrome in women, electrocardiogram load test is positive, but coronary angiography is negative and no coronary artery spasm, the prognosis is good, is considered to be capillary diastolic dysfunction caused by coronary artery system. Myocardial bridge is a usually walk in the connective tissue under the coronary arteries, such as have a walk in the myocardium, its a bunch of myocardial fibers called myocardial bridge. When the heart contracts, can squeeze the artery segments can cause myocardial bridge leading to decreased blood supply of distal myocardial ischemia, combined with the proximal blood vessels often atherosclerotic plaque formation, hence can cause angina. Coronary angiography and intravascular ultrasound can establish the diagnosis.

\section{The cause of coronary heart disease with heart failure}

Coronary heart disease, heart failure are caused by coronary heart disease (CHD) cardiovascular lesions, hardening or blocked, oxygen transport capacity decreased, oxygen to myocardial cells, and the aging of myocardial cell lesion caused by ATP synthesis level drops, not enough energy metabolism in cells, cardiac contractile force is abate. Those cause arrhythmia or heart failure, and a series of concurrent symptoms. Traditional drugs for homodynamic effect, by reducing myocardial oxygen consumption, expanding collateral circulation, increasing coronary blood flow and reducing the peripheral resistance, etc, so as to achieve the purpose of improving ventricular systolic and diastolic function. Its treatment principle mainly by improving the aerobic capacity, rather than the ability to improve the myocardial cell energy metabolism and function, therefore, cannot achieve 
the ideal therapeutic effect. The cause of heart failure, is a primary or secondary cardiomyopathy, cardiomyopathy, myocarditis, coronary heart disease, lung, myocardial infarction, etc. Second, cardiac load excess, divided into excessive pressure load and overload capacity. The former occurs in hypertension, aortic, lung, pulmonary embolism, pulmonary hypertension, etc.; The latter can be found in aortic and mitral insufficiency, pulmonary valve or tricuspid valve closed is not complete, thyroid function hyper function sex heart disease, is from right to left or from left to right shunt congenital heart disease, etc. Third, diastolic limited, found in ventricular diastolic compliance to reduce, such as coronary heart disease, myocardial ischemia, hypertension, myocardial hypertrophy, hypertrophic cardiomyopathy, and so on. On the basis of the basic causes, and then with a cold, fever, activity is excessive, arrhythmia, such as urinary tract infection in the lungs or inducing factors, if not handled in time, will lead to heart failure. After myocardial infarction, cardiac contraction force and comfortable tension were significantly reduced or not harmonious, congestive heart failure gradually appear, can be divided into left heart failure, right heart failure and whole heart failure. Left heart failure symptoms first is difficulty breathing after labor or movement, soon after the rest condition is heavy, a little work, even sleep also feel breathing difficulties, need to rest, half sitting or sitting rear can reduce. Serious resting may also have difficulty breathing, and paroxysmal dyspnea occurred during sleep, at the same time have a cough, cough pink, facial cyanosis, sweating and other symptoms. Right heart failure early symptoms include loss of appetite, abdominal pain, nausea and vomiting. Upper abdomen bag piece and tenderness, jugular vein engorgement, reduced the volume of urine increased, night, double leg edema, severe cases appeared pleural effusion. The whole heart failure can exist at the same time right heart failure and left the signs and symptoms of heart failure, but breathing difficulties can have varying degrees of ease.

The heart failure is to point to in the case of venous return to normal, to reduce cardiac output caused by various kinds of heart disease, can't meet the needs of the state of rest or mild activity when the body's metabolic syndrome. Its etiology is mainly primary myocardial damage and long-term ventricular load is overweight, leading to the decrease in the number of myocardial cells and myocardial diastolic function of the results of the poor. Coronary heart disease (CHD), i.e., short for coronary atherosclerosis sex heart disease, is refers to the coronary artery atherosclerotic vascular cavity block, lead to myocardial ischemia and hypoxia caused by heart disease, and coronary artery functional changes are collectively referred to as coronary heart disease. The disease is a common disease of cardiovascular internal medicine, also is one of the common and frequent diseases in the elderly. Serious when can happen all kinds of complications, such as heart failure, serious damage to life and health of patients.

\section{The treatment of Trimetazidine jointed with Metoprolol in the coronary heart disease with heart failure}

Trimetazidine against epinephrine, norepinephrine, and the effect of vasopressin, can reduce vascular resistance, increase the coronary artery and blood flow, and promote myocardial metabolism and energy production. At the same time could reduce the myocardial oxygen consumption and improve myocardial oxygen supply and demand balance. Also it can increase the tolerance of cardiac glycoside. That may be the function of ischemic myocardium cell protection directly. By saving energy metabolism of ischemia in cells, and prevent the intracellular ATP levels drop, in a stable environment in the cell at the same time ensure that the function of the ion pump to improve and transport through membrane pump normal, reduce intracellular acidosis and prevent the accumulation of the sodium and calcium in the cell protection cell shrinkage capabilities and limitations of oxygen free radicals cytolysis and lining damage. Metoprolol slow resting and exercise heart rate, lower resting and exercise blood pressure. The fast changes in heart rate and heart rate before using this drug have a direct relationship, but has nothing to do with the patient's age. In patients with normal blood pressure or hypertension, weaken the movement the changes of systolic blood pressure, with normal blood pressure, however, does not affect the changes of 
diastolic blood pressure caused by motion. In patients with coronary heart disease, it can better reduce the heart rate and blood pressure during movement and static coupon.

Metoprolol is as class a beta 1-2 receptor blockers, can make the beta 1 - receptor blocking effect, still can make catecholamine blocking contraction of blood vessels, the body's cells and humoral immune damage reduced, myocardial blood supply improvement, cardiac oxygen consumption is reduced, maintenance of cardiac structure and function and the work quickly, oral absorption quickly and completely absorption rate is more than $90 \%$, while the sympathetic and parasympathetic nerve function adjustment and maintenance, the heart rate variation situation improves, to significantly reduce the incidence of sudden death. Trimetazidine is A new type of long chain 3 - ketene coenzyme A sulfur (3 - KAT) inhibitors of enzymes, is pauperizing derivatives, can make the stability of the environment inside the cell, the cell avoid dissolve, lining from injury, acidosis caused by the accumulation of calcium and sodium reduce greatly, reduce the release of oxygen free radicals and endothelia, potassium sodium pump to ensure the normal operation, the cardiac load decreases, and alleviate hypoxia on myocardial cell damage. Through perfecting the mitochondrial energy metabolism directly improve myocardial function. Makes the heart in the case of ischemia hypoxia, improve metabolism, provide more energy for myocardial.

The flow of blood to the heart is normal, $60 \% \sim 80 \%$ of myocardial ATP from fatty acid oxidation, but from the point of view of oxygen consumption, the effectiveness of the direct oxidation of glucose for energy is better than that of fatty acid oxidation. Trimetazidine is 3 - keto coenzyme sulfur (3 - KAT) inhibitors of enzymes, 3 - KAT within the direct inhibition of mitochondrial activity, which inhibits fatty acid oxidation process and reduce myocardial fatty acid intake, improve myocardial glucose in the oxidation process. Produce the same amount of ATP, compared with the fatty acid oxidation, glucose of oxygen consumption of fatty acids that are lower than $10 \%$, can guarantee under the anoxic condition, to provide more energy for myocardial, strengthening the cardiac function recovery, myocardial contraction force. Trimetazidine can also ease of intracellular acidosis caused by ischemia, strengthen the activity of the mitochondria, inhibit free radical levels of endothelia release, avoid the happening of lyses and lining damage, and reduce because of myocardial cell apoptosis and necrosis caused by decreased ventricular remodeling and cardiac function.

\section{Conclusion}

General speaking, in patients with coronary heart disease with heart failure on the basis of conventional treatment with Trimetazidine joint with Metoprolol, the function and effect is very good, and is worthy of clinical popularization and application. Trimetazidine combined with Metoprolol can effectively improve cardiac function in patients with coronary heart disease, heart failure, and protect the heart, effectively optimize the myocardial cell energy metabolism, improve the patient's heart function and sports endurance, significantly improve the clinical symptoms, and is worth clinical promotion.

\section{Acknowledgement}

This research was financially supported by the National Science Foundation.

\section{References}

[1] Kwan Keung Leung, Juan Liang, Man Ting Ma, Po Sing Leung. Angiotensin II Type 2 Receptor Is Critical for the Development of Human Fetal Pancreatic Progenitor Cells into Islet like Cell Clusters and Their Potential for Transplantation[J]. STEM CELLS. 2012 (3)

[2] Santosh G. Menon, Roger M. Mills, Ute Schellenberger, Syed Saqhir, Andrew A. Protter. Clinical Implications of Defective B - Type Natriuretic Peptide[J]. Clin Cardiol. 2009 (12)

[3] Andrew J.Burger. A Review of the Renal and Neurohormonal Effects of B - Type Natriuretic Peptide[J]. Congestive Heart Failure. 2012 (1) 
[4] W. Franklin Peacock,Charles L. Emerman,Marc A. Silver. Nesiritide added to standard care favorably reduces systolic blood pressure compared with standard care alone in patients with acute decompensated heart failure[J]. American Journal of Emergency Medicine. 2012 (3)

[5] William T. Abraham, Kirkwood F. Adams, Gregg C. Fonarow, Maria Rosa Costanzo, Robert L. Berkowitz, Thierry H. LeJemtel,Mei L. Cheng,Janet Wynne. In-Hospital Mortality in Patients With Acute Decompensated Heart Failure Requiring Intravenous Vasoactive Medications[J]. Journal of the American College of Cardiology. 2012(1)

[6] Rika Kawakami, Yoshihiko Saito, Ichiro Kishimoto, Masaki Harada, Koichiro Kuwahara, Nobuki Takahashi, Yasuaki Nakagawa, Michio Nakanishi, Keiji Tanimoto, Satoru Usami, Shinji Yasuno, Hideyuki Kinoshita, Hideki Chusho, Naohisa Tamura, Yoshihiro Ogawa, Kazuwa Nakao. Overexpression of Brain Natriuretic Peptide Facilitates Neutrophil Infiltration and Cardiac Matrix Metalloproteinase-9 Expression After Acute Myocardial Infarction[J]. Circulation. 2014 (21)

[7] Damien Logeart, Gabriel Thabut, Patrick Jourdain, Christophe Chavelas, Pascale Beyne, Florence Beauvais, Erik Bouvier, Alain Cohen Solal. Predischarge B-type natriuretic peptide assay for identifying patients at high risk of re-admission after decompensated heart failure[J]. Journal of the American College of Cardiology . 2014 (4)

[8] Ann M. Kapoun, Faquan Liang, Gilbert O’Young, Deborah L. Damm, Diana Quon,R. Tyler White,Kimberly Munson, Andrew Lam, George F. Schreiner,Andrew A. Protter. B-Type Natriuretic Peptide Exerts Broad Functional Opposition to Transforming Growth Factor- $\beta$ in Primary Human Cardiac Fibroblasts: Fibrosis, Myofibroblast Conversion, Proliferation, and Inflammation[J]. Circulation Research: Journal of the American Heart Association . 2014 (4)

[9] H.Krum, D.Liew. New and emerging drug therapies for the management of acute heart failure[J]. Internal Medicine Journal . 2013 (11)

[10] Hassan M.E. Azzazy,Robert H. Christenson. B-Type Natriuretic Peptide: Physiologic Role and Assay Characteristics[J]. Heart Failure Reviews. 2013 (4) 